\title{
Anthropometric and inflammatory status in west Algerians adolescents
}

\author{
H. Ghomari-Boukhatem ${ }^{1}$, A. Bouchouicha ${ }^{1}, \mathrm{~K}$. Mekki $^{1}$ and M. Bouchenak ${ }^{1}$ \\ ${ }^{1}$ Laboratoire de Nutrition Clinique et Métabolique, Faculté des Sciences, Université dOran, Algérie
}

Obesity in adolescence constitutes an important public health problem that has many short- and long-term effects. While clinical symptoms are not observed until adulthood, atherosclerotic lesions may form during younger years. There is strong evidence that inflammation plays a key role in atherosclerosis development. In Algerian children and adolescents, the increased prevalence of overweight and obesity was noted and related with high energetic intake.

The aim of this study was to evaluate some inflammatory markers in adolescents. A study was carried out in 400 collegians in Oran aged to 10-17 years (sex ratio girl/boy $=180 / 220$ ) divided into 4 groups according to the IOTF (2005) classification. Serum Tumor Necrosis Factor (TNF- $\alpha$ ), interleukin-1 $\beta$ (IL-1 $\gamma$ ), interleukin-6 (IL-6) and C-reactive protein (CRP) were analyzed by enzyme immunoassay (EIA) (Cayman Chemical Kits).

The anthropometric parameters showed that $16 \%$ of adolescents were lean, $66 \%$ were normal, $13 \%$ of them were overweighed and $5 \%$ obese. Body mass index, leptin, TNF- $\alpha$ and CRP values were higher in obese and overweight adolescents compared to those with normal weight $(p<0.05)$, meanwhile adiponectin levels were lower in the adolescents with obesity and overweight. There were no significant differences in IL-1 $\beta$ and IL-6 between groups.

Overweight and obesity are associated with elevated inflammation markers. These adolescents are at high risk of cardiovascular diseases, thus requiring a nutritional intervention. 